\title{
Butterworth filters as tracking differentiators
}

This paper was downloaded from TechRxiv (https://www.techrxiv.org).

\section{LICENSE}

CC BY-SA 4.0

SUBMISSION DATE / POSTED DATE

$27-01-2021 / 28-01-2021$

CITATION

olivier, philip (2021): Butterworth filters as tracking differentiators. TechRxiv. Preprint. https://doi.org/10.36227/techrxiv.13646678.v1

$\mathrm{DOI}$

10.36227/techrxiv.13646678.v1 


\section{Butterworth filters as tracking differentiators}

\section{P. D. Olivier}

This letter describes how traditional Butterworth low pass filters can enhance the performance of the tracking differentiator introduced by Han by mitigating the effect of additive high frequency noise that corrupts the output measurement. The tracking differentiator obtains much of its utility from its realization in cascaded integral form. By combining the cascaded integral form realization of Butterworth low pass filters with its the noise rejection features one can design a tracking differentiator that is efficiently tuned to reject high frequency output noise.

Introduction: Han, in [1], introduced (at least to English speaking audiences) his tracking differentiator, TD. The tracking differentiator not only tracks an input function, but also tracks its derivative(s) without explicitly computing the derivative, thereby avoiding many well-known problems. In [1], Han constructed a tracking differentiator from a simple low pass filter of the form $(0<\tau \ll 1)$

$$
s \widehat{U}=s F(s) U, \quad F(s)=\frac{1}{(\tau s+1)^{2}}=\frac{1 / \tau^{2}}{s^{2}+\frac{2}{\tau} s+\frac{1}{\tau^{2}}}
$$

This filter $\mathrm{F}(\mathrm{s})$ can be realized in the cascaded integral form

Where

$$
\frac{d}{d t}\left[\begin{array}{l}
x_{1} \\
x_{2}
\end{array}\right]=\left[\begin{array}{cc}
0 & 1 \\
-1 / \tau^{2} & -2 / \tau
\end{array}\right]\left[\begin{array}{l}
x_{1} \\
x_{2}
\end{array}\right]+\left[\begin{array}{c}
0 \\
1 / \tau^{2}
\end{array}\right] u
$$

The arrow is used to indicate "tracking".

A similar idea is used by $\mathrm{Lu}$ et al in [2] where a tracking differentiator (this term not used) with the low pass transfer function

$$
F(s)=\frac{\omega_{1} \omega_{2}^{2}}{\left(s+\omega_{1}\right)\left(s^{2}+2 \zeta \omega_{2} s+\omega_{2}^{2}\right)}
$$

With cascade integral realization

$$
\begin{aligned}
& \dot{x}_{1}=x_{2} \\
& \dot{x}_{2}=x_{3} \\
& \dot{x}_{3}=\omega_{1} \omega_{2}^{2} x_{1}-\left(\omega_{2}^{2}-2 \zeta \omega_{2}\right) x_{2}-\left(2 \zeta \omega_{2}+\omega_{1}\right) x_{3}+\omega_{1} \omega_{2}^{2} u
\end{aligned}
$$$$
\text { Where } x_{1}=\hat{u}, x_{2}=\hat{\dot{u}}, x_{3}=\hat{u} \text {. }
$$

In both cases the tracking differentiator is based on a low pass filter realized in cascade integral form. The contribution of this paper is to suggest using cascade integral realization based on the traditional Butterworth low pass filter to facilitate the design of tracking differentiators. Obviously, any cascade integral realization of a filter that rejects the noise would work. Unfortunately, Chebyshev, and elliptic filters do not have a suitable cascade integral realizations.

The rest of this letter is organized as follows: how to use a standard low pass filter as a tracking differentiator (TD); an example using a $3^{\text {rd }}$ order Butterworth TD; A $2^{\text {nd }}$ order Butterworth TD is applied to the speed control of a series-wound DC motor without output measurement noise and with output measurement noise; cascade integrator realizations are reviewed; a review of Butterworth transfer functions; finally conclusions are presented.

Using Butterworth filters as tracking differentiators: The main idea behind this letter is to replace the low pass filters suggested by Han and /or Lu et al with the more traditional Butterworth low pass filters while retaining the realization of the filters in cascaded integral form.

The advantages of using the Butterworth low pass filters include:

1) They have a cascade integral realization. This allows the signal as well as some of its derivatives to by estimated (tracked) without explicitly computing the derivatives.

2) they are easily tuned to achieve "maximally flat and high" low frequency response which provides faster tracking and maximally low high frequency response to reject the corrupting noise.

3) the filter order can be chosen to achieve sharp transition from the low frequency region to the high frequency region.
Perusal of the Butterworth transfer functions confirms that they all have transfer functions of the form

$$
F(s)=\frac{a_{0}}{s^{n}+a_{n-1} s^{n-1}+\cdots+a_{1} s+a_{0}}
$$

The second and third order Butterworth filter transfer functions are $(S=$ $s / \omega_{0}$, where $\omega_{0}$ is the cutoff (or half power) frequency, see any table of Butterworth transfer functions, these were taken from Wikipedia)

$$
\begin{gathered}
B_{2}(S)=\frac{1}{S^{2}+1.414 S+1} \\
B_{3}(S)=\frac{1}{(S+1)\left(S^{2}+S+1\right)}
\end{gathered}
$$

The cascaded integral state-space realization of the $\mathrm{n}^{\text {th }}$ order filter is

$$
\frac{d}{d t}\left[\begin{array}{c}
x_{1} \\
x_{2} \\
x_{3} \\
\vdots \\
x_{n-1} \\
x_{n}
\end{array}\right]=\left[\begin{array}{cccccc}
0 & 1 & 0 & \cdots & 0 & 0 \\
0 & 0 & 1 & \cdots & 0 & 0 \\
0 & 0 & 0 & \cdots & 0 & 0 \\
\vdots & \vdots & \vdots & \ddots & \vdots & \vdots \\
0 & 0 & 0 & \cdots & 0 & 1 \\
-a_{0} & -a_{1} & -a_{2} & \cdots & -a_{n-2} & -a_{n-1}
\end{array}\right]\left[\begin{array}{c}
x_{1} \\
x_{2} \\
x_{3} \\
\vdots \\
x_{n-1} \\
x_{n}
\end{array}\right]+\left[\begin{array}{c}
0 \\
0 \\
0 \\
\vdots \\
0 \\
a_{0}
\end{array}\right] u
$$

Where

$$
\left[\begin{array}{c}
x_{1} \\
x_{2} \\
x_{3} \\
\vdots \\
x_{n-1} \\
x_{n}
\end{array}\right]=\left[\begin{array}{c}
\hat{u} \\
\hat{\hat{u}} \\
\dot{\hat{u}} \\
\vdots \\
\hat{u}^{[n-2]} \\
\hat{u}^{[n-1]}
\end{array}\right] \rightarrow\left[\begin{array}{c}
u \\
\dot{u} \\
\ddot{u} \\
\vdots \\
u^{[n-2]} \\
u^{[n-1]}
\end{array}\right]
$$

The claim that $\hat{u}$ tracks $u$ (and $\dot{\hat{u}}$ tracks $\dot{u}$, etc.) for sufficiently slowly varying $u$ 's relative to the bandwidth of the low pass filter follows from

a) the low pass characteristics of the filter and the Final Value Theorem,

b) that $\mathrm{F}(0)=1$,

c) the cascaded integral form of the state-space representation, i.e. $\dot{x}_{i}=x_{i+1}$ for $i=1, \cdots, n-1$.

The high frequency noise rejection property follows from the fact that the tracking differentiator is a realization of a well understood low pass filter.

A $3^{\text {rd }}$ order Butterworth TD: The performance of a $3^{\text {rd }}$ order Butterworth filter is investigated. The uncorrupted signal to be tracked is

$$
u(t)=1-e^{-t}
$$

This signal is corrupted by noise of the form

$$
n(t)=0.1 \sin (100 t)
$$

Using the $3^{\text {rd }}$ order Butterworth filter transfer function with a cut off frequency of $10 \mathrm{rad} / \mathrm{sec}$ found in a subsequent section. The simulation results are shown in Figure 1.

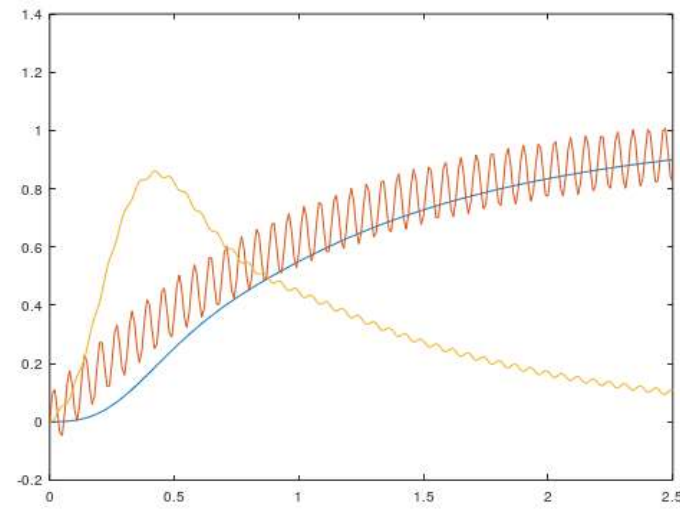

Figure 1. Simulation results for $3^{\text {rd }}$ order Butterworth filter with cut off frequency 10 used as a Tracking Differentiator.

Sinusoidal noise with amplitude 0.1 and frequency 100 .

1. The red curve is the corrupted input, $u(t)+n(t)$

2. The blue curve is the filtered corrupted input signal

3. The orange curve is the derivative of the filtered corrupted input signal

4. The unfiltered derivative has a sinusoidal term with amplitude 10 so it is not shown in this figure. 
A higher cut off frequency will improve the tracking response, but reduce the noise rejection. Using a higher order filter with the same cut off frequency will marginally improve both tracking response (keep low frequency gain high over a larger frequency range) and noise rejection (cause high frequency gain to approach zero faster).

$2^{\text {nd }}$ order Butterworth TD applied to a series-wound DC motor: The series-wound DC motor considered by Khoroshun in [3] is used to demonstrate the use of the Butterworth TDs. First the case of noise free output measurement is considered. Then the output is assumed to be corrupted. A feedback controller is implemented using the output of the TD.

Consider a feedback system consisting of the following components

1) The (possibly nonlinear) plant, with generic input $u$ (in this case $V$ ) and generic output $y$ (in this case $\omega$ ).

2) The error computed as $e=r-\omega$ where $r$ is the reference input.

3) An augmented TD with input $e$ and outputs, $\hat{e}, \dot{\hat{e}}, \ddot{\hat{e}}, \cdots$ and $\int_{0}^{t} \hat{e}(\tau) d \tau$. And

4) A PID (or more complicated) controller, with inputs from the augmented TD and output $V$ which completes the feedback loop.

The model of the series-wound DC motor used in these examples is

$$
\frac{d}{d t}\left[\begin{array}{l}
i \\
\omega
\end{array}\right]=\left[\begin{array}{c}
-78.505 i-1.3477 i \omega \\
175.42 i^{2}-6.2312 \omega
\end{array}\right]+\left[\begin{array}{c}
10.903 \\
0
\end{array}\right] V
$$

The numerical values come from the motor parameters used in [3], $\mathrm{V}$ is the input voltage, and the measured output is $\omega$.

The desired steady-state speed is $r=\bar{\omega}_{d}=2$ revolutions per second $=12.57 \mathrm{radians} / \mathrm{sec}$. The error is $e=\bar{\omega}_{d}-\omega$. In the presence of noise, the filtered estimate produced by the TD, $\hat{e}$, should be used

The augmented $2^{\text {nd }}$ order Butterworth TD is

$$
\frac{d}{d t}\left[\begin{array}{c}
\int_{0}^{t} \hat{e}(\tau) d \tau \\
\hat{e}(t) \\
\dot{\hat{e}}(t)
\end{array}\right]=\frac{d}{d t}\left[\begin{array}{l}
z_{1} \\
z_{2} \\
z_{3}
\end{array}\right]=\left[\begin{array}{ccc}
0 & 1 & 0 \\
0 & 0 & 1 \\
0 & -\omega_{0}^{2} & -1.414 \omega_{0}
\end{array}\right]\left[\begin{array}{c}
Z_{1} \\
z_{2} \\
z_{3}
\end{array}\right]+\left[\begin{array}{c}
0 \\
0 \\
\omega_{0}^{2}
\end{array}\right] e
$$

Using the states of the augmented TD, a proportional $(\boldsymbol{P})$ controller has the form $V=K_{P} \hat{e}=K_{P} z_{2}$. A proportional-derivative (PD) controller has the form $V=K_{P} \hat{e}+K_{D} \dot{\hat{e}}=K_{P} Z_{2}+K_{D} Z_{3}$. A proportional-integral-derivative (PID) controller has the form $V=$ $K_{P} \hat{e}+K_{I} \int_{0}^{t} \hat{e} d \tau+K_{D} \dot{\hat{e}}=K_{P} Z_{2}+K_{I} z_{1}+K_{D} Z_{3}$. Additional derivatives could be added for more complicated controllers.

PI controller without noise. Figure 2 shows the performance of the series-wound DC motor using a PI controller with $\mathrm{K}_{\mathrm{P}}=\mathrm{K}_{\mathrm{I}}=0.2$ with no noise, using a Butterworth TD with $\omega_{0}=15$. The reference input is $\omega_{d}=12.57 \mathrm{rad} / \mathrm{sec},(2$ revolutions $/ \mathrm{sec})$.

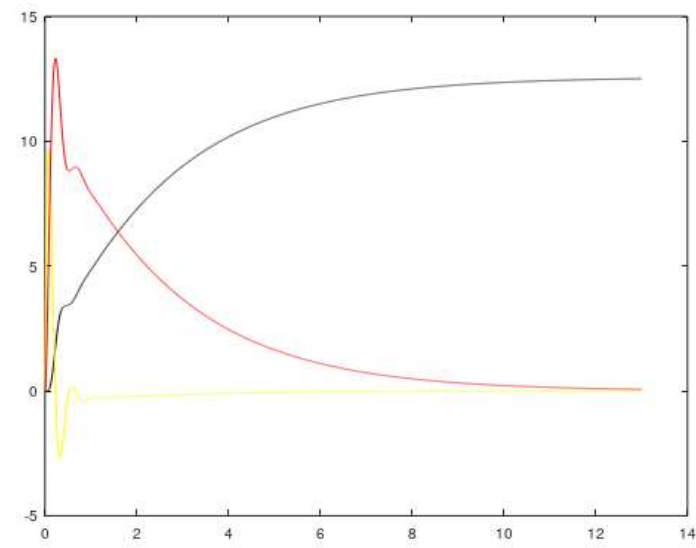

Figure 2. The black curve is $\omega(t)$; the red curve is the TD estimate of the tracking error; the yellow curve is one tenth of the TD estimate of the derivative of the tracking error.
PI controller with noise. Figure 3 shows the performance of the series-wound DC motor using a PI controller with $\mathrm{K}_{\mathrm{P}}=\mathrm{K}_{\mathrm{I}}=0.2$ with noise, using a Butterworth TD with $\omega_{0}=15$. The reference input is $\omega_{d}=12.57 \mathrm{rad} / \mathrm{sec},(2$ revolutions $/ \mathrm{sec})$. In addition, the noise is chosen to be $n(t)=10 \sin (366 t)$.

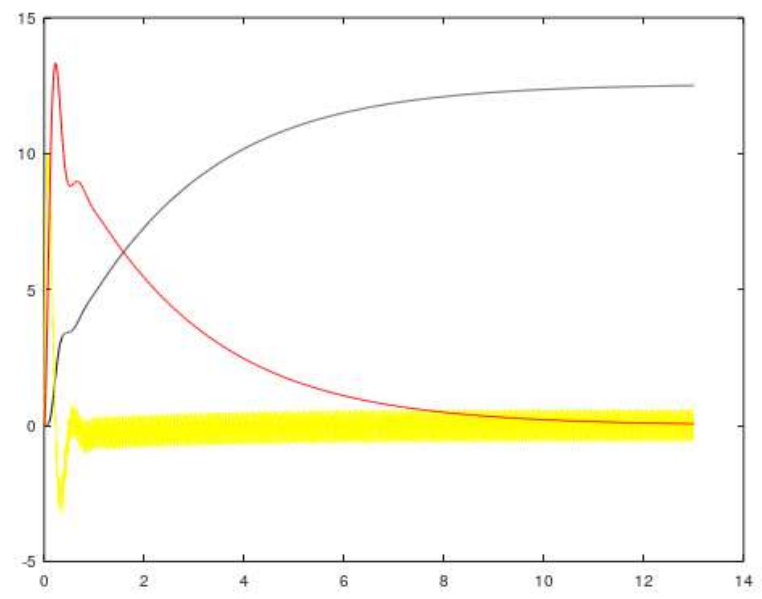

Figure 3. The black curve is $\omega(t)$; the red curve is the TD estimate of the tracking error; the yellow curve is one tenth of the TD estimate of the derivative of the tracking error.

Conclusions. As these examples verify, using the estimates of the error and the integral of error produced by the TD based on Butterworth low pass filters perform quite well. Comparing the performance with noise and without noise indicates that the noise rejection capability is quite good.

Tracking differentiators that reject high frequency noise can be built from any stable transfer function that has the following characteristics:

1. It can be realized in cascaded integral form (the numerator is a constant)

2. It is a low pass filter

3. The DC value of the transfer function is 1 .

The Butterworth filter low pass transfer function is perfect for this task. Other common low pass filters (Chebyshev, elliptic, etc) have numerators that are non-constant polynomials and hence cannot be realized in cascade integral form.

The real attractiveness of using Butterworth filters as TDs stems from the fact that it is easy to set the order of the filter and its half power frequency to control both the passband and the steepness of frequency response in the transition region.

P. D. Olivier, PhD, PE, Engineering Analysis and Design, Salt Lake City, Utah.

E-mail: Philip.d.olivier@gmail.com

\section{References}

1. Han, J.: 'From PID to Active Disturbance Rejection Control', IEEE Transactions on Industrial Electronics, 2009, 56, (3), pp. 900-906, doi: 10.1109/TIE.2008.20111621.

2 Lu, P., Sandy, T., Buchli, J., 'Nonlinear disturbance attenuation control of hydraulic robotics,' arXiv:1808.01445v1 [cs.RO] 4 Aug 2018.

3 Khoroshun, A.S., 'Stability and speed control of a series-wound DC motor', International Applied Mechanics, Vol. 52, No. 4, July 2016 doi: 10.1007/s10778-016-0768-0. 